\title{
The contribution of Marie Skłodowska-Curie to the development of modern oncology
}

\author{
Andrzej Kułakowski
}

Published online: 18 February 2011

(C) The Author(s) 2011. This article is published with open access at Springerlink.com

At the end of 19 th century a few fundamental discoveries changed diagnostic and therapeutic possibilities in medicine and, particularly, in oncology: in 1895 Wilhelm Roentgen from Germany discovered X-rays, in 1886 Henry Becquerel described the phenomenon of radioactivity of uranium, and in 1898 Marie and Pierre Curie discovered radium and polonium. In 1903 the Nobel Prize for Physics was awarded jointly to Henry Becquerel, and Marie and Pierre Curie for the discovery of radioactivity. Maria SkłodowskaCurie received the 1911 Nobel Prize for Chemistry for her discovery of radium and polonium (Fig. 1).

The discovery of X-rays by Roentgen was a turning point in diagnostics. It enabled precise evaluation of internal organs hitherto completely inaccessible for investigation. First, it became possible to visualize the bones (one of the first X-rays in history was an X-ray of the hand of Roentgen's wife; Fig. 2); then, after introduction of various contrast media (barium meal, iodine preparations) other organs could also be visualized: the alimentary tract, biliary routes, and blood vessels.

Even now radiology is an essential tool in modern diagnosis and, sometimes, in the treatment of many diseases. Modern techniques are, of course, more precise and sophisticated. Computer analysis of X-ray images has enabled the introduction of computer tomography (CT) scans, which give a very precise view of the internal organs inaccessible with classical methods. It is interesting to

Published in the special issue Radioanalytics - Dedicated to Marie Sklodowska-Curie with Guest Editors Boguslaw Buszewski and Philippe Garrigues

A. Kułakowski $(\triangle)$

The Society in Tribute to Maria Skłodowska-Curie,

15 Wawelska Str,

02-061 Warsaw, Poland

e-mail: AKulakowski@vp.pl know that to obtain a simple X-ray in the 1890 s required more than 30 minutes of exposure. Despite further sophistication of the equipment, for example helix CT scan and NMR (nuclear magnetic resonance), the discovery of $\mathrm{X}$-rays remains the basis of the technique.

Natural radioactivity, discovered by Becquerel and Marie and Pierre Curie at the same time, led to new therapeutic and diagnostic methods in medicine. First clinical applications of radioactive substances were undertaken very soon after. As early as 1899 Tage Sjoergren from Sweden reported the first case of a malignant tumor of the skin cured by use of radioactive source application.

Kassabian's Medical Manual issued in 1907 in Philadelphia gives a list of tumors treated by radiotherapy. Many of these are still treated with this method. Nowadays the indications are more precise and the equipment is much more sophisticated. One should also note that at that time radiotherapy was also recommended in cases completely unjustified according to our present knowledge, e.g. varicose veins, epilepsy, acne, and others. However, even at the time doctors observed harmful side effects of radiotherapy, although first reports were rather anecdotal: in July 1896 newspapers described the case of someone who wanted to investigate, on himself, effect of radioactivity on the skull and the brain - as result he lost all his hair.

Discovery of radium and polonium caused rapid and, initially, completely uncontrolled use of radium in all fields of life and medicine. The absence of proper dosimetric methods and proper safety measures resulted in many complications very often fatal-Marie Skłodowska-Curie experienced this herself. Gaining experience in the side effects resulted in elaboration of the modern principles of radiotherapy. Today's radiotherapy is an essential therapeutic method in oncology.

During the first 50 years external beam radiation was based on the use of radium and X-rays. Now we use cobalt 


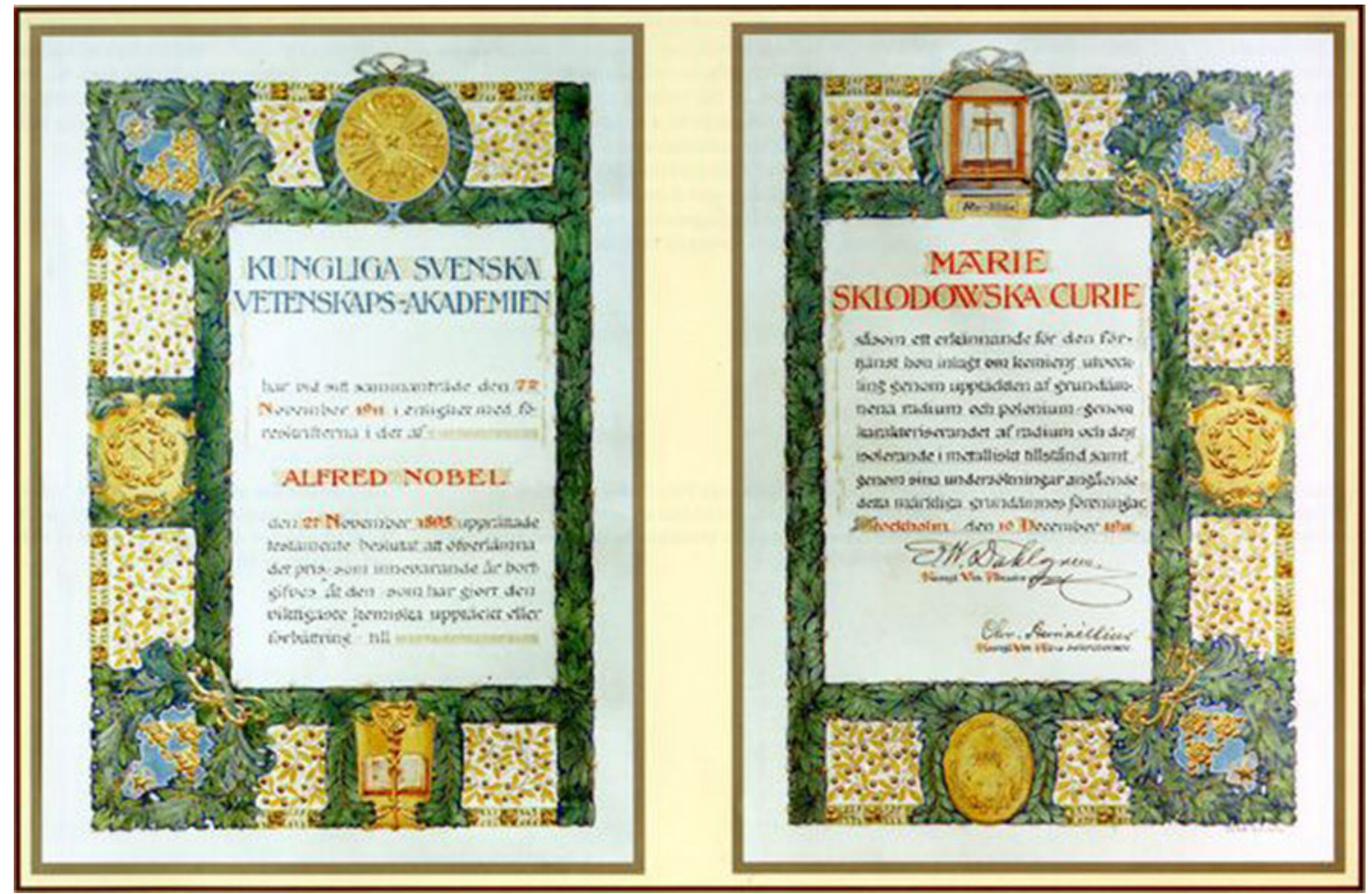

Fig. 1 The 1911 Nobel Prize for Chemistry for Maria Skłodowska-Curie’s discovery of radium and polonium

bomb and linear accelerators - equipment which is completely safe for the patient and medical staff. Introduction of computers has enabled proper treatment planning, precise dosage, and minimal impairment of surrounding normal tissues.

We can see also dynamic progress in another field of radiotherapy i.e. brachytherapy. Greek term "brachy" means "near" - in this case the radioactive source is in direct contact with the tissue of the tumor. The first report of effectively treatment with this method of basal cell carcinoma of the skin was published in 1904 (Goldberg and London). In 1904 a diagram showing the effect of a magnetic field on the radium rays was published by Cleaves. He gives the following commentary "The beta rays are bent strongly to the right, the gamma rays as are the x-rays are not bent at all, and the alpha rays are bent very little to the left." Earlier Pierre Curie demonstrated that at least two different kinds of rays were emitted from radioactive substances. Radium, discovered by MarieSkłodowska-Curie, was first used in brachytherapy. At the very beginning it was placed directly on the surface of the tumor or intracavitary (in cancer of the vagina or uterus). Very soon, as early as the $1910 \mathrm{~s}$, doctors started to introduce the radioactive source interstitially, i.e. deep into the tumor. Cancers of the breast, prostate, esophagus, and brain were among the first treated by this method. Because of a lack of proper dosimetry and the inevitability of direct contact with the radioactive material, the method was very dangerous for the doctors who used it. The method was substantially improved by use of radioactive isotopes with the afterloading method. Cesium and iridium are new isotopes in use. Modern computer software enables very precise elaboration of effective and, simultaneously, safe dosage; this is particularly important with increasing use of high doses (HDR - high dose rate) in brachytherapy. Brachytherapy is successfully used for treatment of cancer of the genitourinary tract, head and neck cancers, and carcinoma of the penis. In many cases its effectiveness is similar to radical but sometimes mutilating surgery. In advanced incurable cancer, brachytherapy can be used for palliation, giving relief to the patient and offering some comfort during the last months of his/her life. New diagnostic and interventional methods in endoscopy and radiology enable the use of various applicators without the need for difficult and dangerous surgical intervention, particularly for patients in poor condition. Brachytherapy is very effective in palliative treatment of lung cancer, esophageal cancer, and tumor of alimentary tract.

A relatively new technique based on the discoveries of Marie Skłodowska-Curie is nuclear medicine which uses substances labeled with radioisotopes introduced into the organs of the patient for imaging of the tumors. Progress in nuclear medicine was possible after the Second World War when, after the discoveries of Frederic and Irene JoliotCurie from 1930s, it became possible to produce artificial 


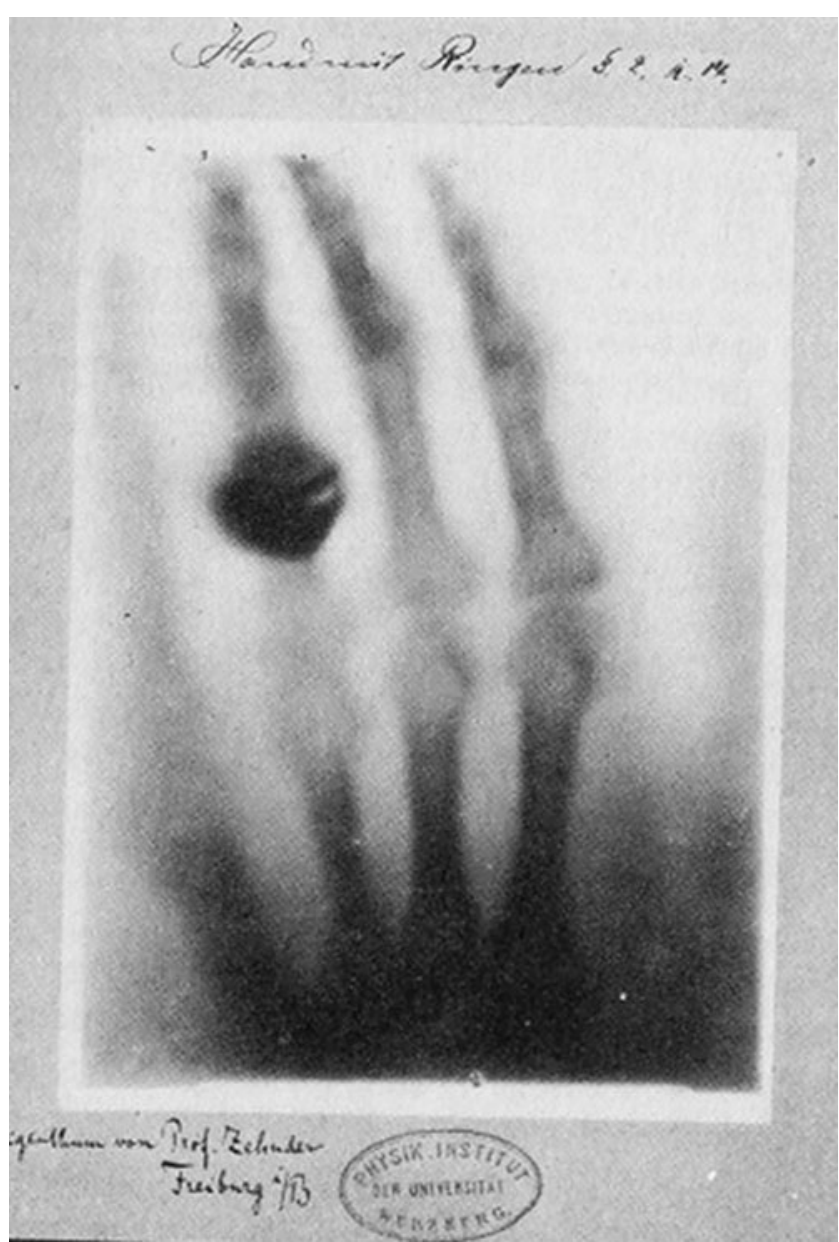

Fig. 2 The first X-ray in history - the hand of Konrad Roentgen's wife

radioisotopes in amounts suitable for use in medicine (Fig. 3).

Radioactive isotopes of such elements as iodine, indium, or technetium with relatively short half-lives can be used in both diagnosis and therapy. They are being introduced into

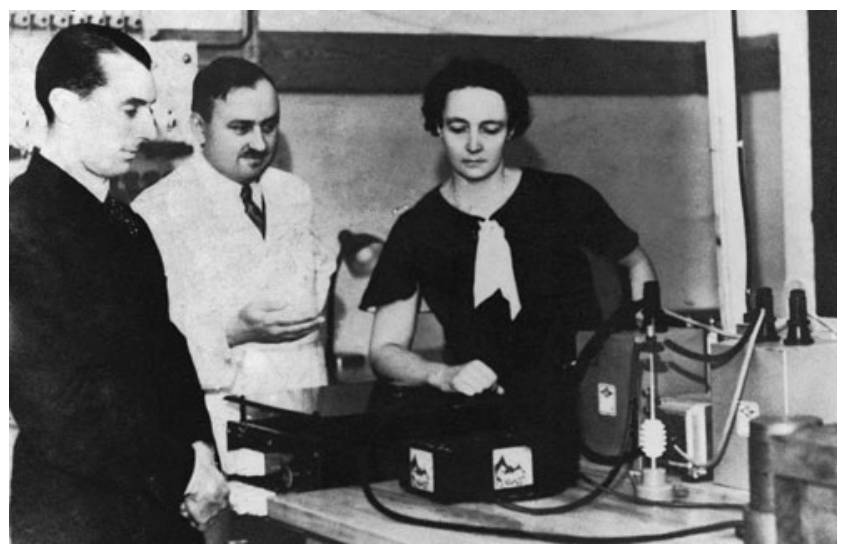

Fig. 3 Frederic and Irene Joliot-Curie organs in combination with other substances which selectively bind to various tissues or tissue fluids. Reading of the distribution of the radioactivity by use of gamma-cameras, which substituted popular Geiger counters, enables location of the cancer tissue or detection of the direction of lymphatic flow. This is very often helpful to the oncologist when choosing the proper tactics for treatment. Enormous progress has been made during recent years in the construction of devices giving a more precise view of the organs investigated, for example SPECT (single photon emission computed tomography) or, even better, PET (positron emission tomography). A new device with increasing possibilities for the surgeon is an intraoperative detector of gamma radiation. The probe enables more precise location of tumor tissue previously labeled with an isotope and makes possible more radical procedures or, on the other hand, enables evaluation of the inoperability of the tumor. A new possibility for surgical removal of tumors is RIGS-radioimmunoguided surgery.

The most urgent problem at the moment is elaboration of substances which specifically bind to neoplasmatic tissue. Progress here is significant, mostly because of the production of better monoclonal antibodies. Nuclear medicine also uses radioisotopes for treatment of cancer-by using selective binding of iodine to thyroid tissue we can destroy residual microscopic fragments of cancer tissue left after surgery. At the moment this is only experimental, but maybe other tumors will soon be treated by use of monoclonal antibodies in clinical practice.

Cancer is still very often a mortal disease and if it is cured patients suffer very much during the treatment and sometimes become disabled at the cost of being alive. However progress in oncology is visible, and with still improving methods of early diagnosis and better therapeutic methods the number of cured patients is still increasing.

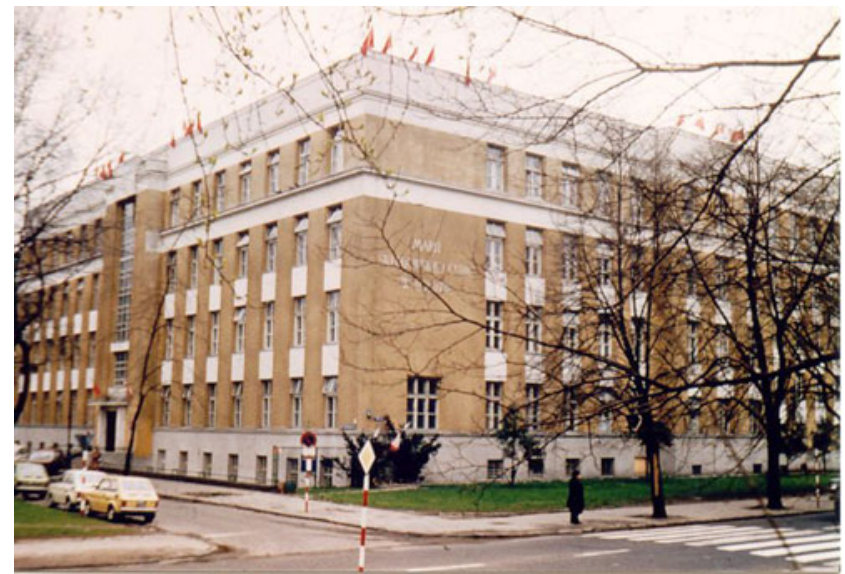

Fig. 4 The Marie Skłodowska-Curie Memorial Cancer Center in Warsaw 


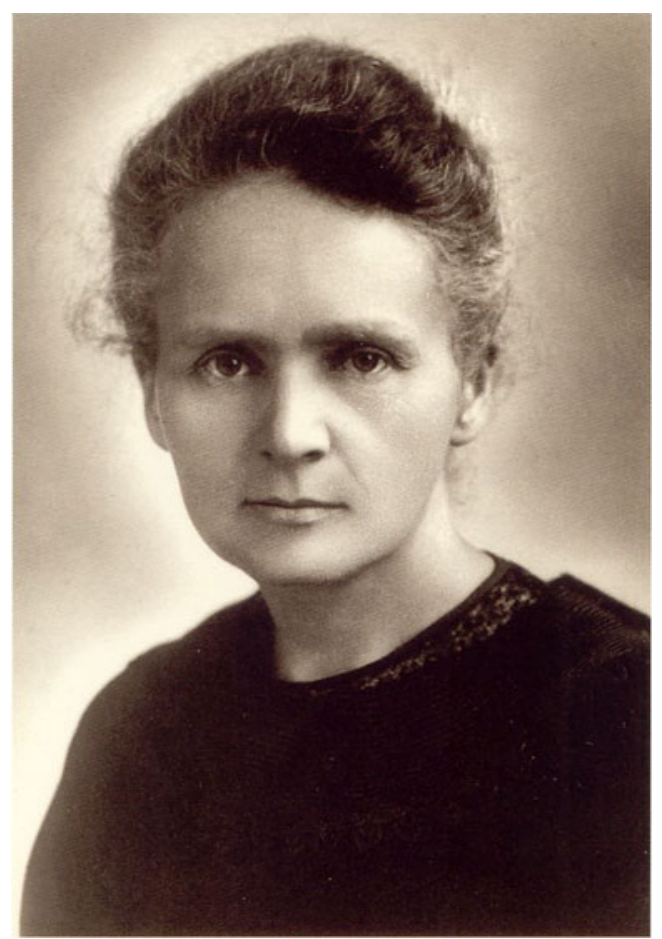

Fig. 5 Marie Skłodowska-Curie

Discoveries made 100 years ago are still the basis for very important scientific investigations giving hope, and the possibility of saving many people. Marie SkłodowskaCurie was not a doctor but she recognized the value of her discoveries for medicine. The Institute Curie in Paris and the Radium Institute in Warsaw (now Marie SkłodowskaCurie Memorial Cancer Center) were founded on the basis of her inspiration (Fig. 4).

The activity of the Marie Skłodowska-Curie Memorial Cancer Center includes not only the treatment of the patients but, as Marie Curie wanted, research also. Every clinical specialist should keep in mind that progress in clinical medicine and every-day medical practice cannot exist without progress in research. Marie Curie said: "Therapeutic activity in such new field requires strong base which is constituted by physical and chemistry studies of new substances, if the base does not exist, theory becomes just empiricism and routine use of indiscriminately popular methods in which there are, sometimes fundamental, mistakes" (Fig. 5).

"Therapy should be permanently backed up by scientific research without which no progress is possible. Moreover, the search for pure knowledge is one of the important needs of mankind." (From the welcoming speech of Marie Skłodowska-Curie at the opening of Radium Institute in Warsaw 29 May 1932.)

Open Access This article is distributed under the terms of the Creative Commons Attribution Noncommercial License which permits any noncommercial use, distribution, and reproduction in any medium, provided the original author(s) and source are credited.

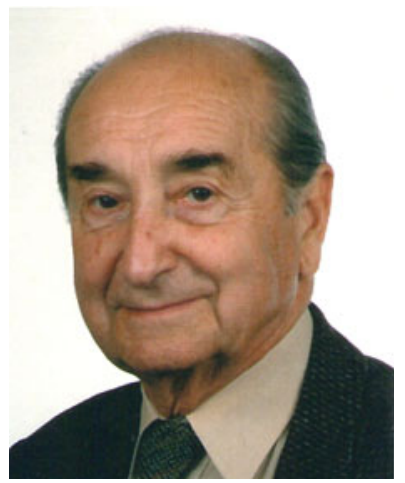

\section{Andrzej Kulakowski}

has been Professor Emeritus of oncologcial surgery since 1999. Before his retirement he was Professor of oncological surgery and Director General of the Maria Skłodowska-Curie Memorial Cancer Center and Institute of Oncology. He is one of the founders of "The Society in Tribute to Maria SkłodowskaCurie" and was its president from 1995-2010. He is also founder of the Polish Society of Surgical Oncology. He is author of numerous publications and has received many distinguished awards, for example the Medicus Magnus and Honorary Membership of the American College of Radiology. He is currently a consultant for surgical oncology at the Holycross Cancer Center in Kielce, Poland. 